\title{
THE NATURAL HISTORY OF THE FUNGUS GNATS LEPTOMORPHUS BIFASCIATUS (SAY) AND L. SUBCAERULEUS (COQUILLETT)
} (DIPTERA: MYCETOPHILIDAE) ${ }^{1}$

\author{
By William G. Eberhard \\ Museum of Comparative Zoology, Harvard University ${ }^{2}$
}

Although there are many species of Mycetophilidae (or Fungivoridae) and some that are quite common, little is known about the natural history of most species. The literature on the genus Leptomorphus is typically scanty: there are no descriptions of any activities of adults, and only brief descriptions of the general habitat and activities of eggs, larvae, and pupae of one species, L. walkeri (Edwards 1925, Brocher 1931, Madwar 1937). This report, which includes observations on all life stages of Leptomorphus bifasciatus and L. subcaeruleus, is the first study of the natural history of these species.

Most of the observations were made during the summer of 1968 near Rensselaerville, N.Y., at the E. N. Huyck Preserve, and a few additional notes were made on individuals found near West Chesterfield, New Hampshire. Leptomorphus subcaeruleus was found near West Chesterfield, and both this species and L. bifasciatus (with L. subcaeruleus most common) occurred on the Huyck Preserve. I discerned no difference in the habits of the two species, and adult males and females of both species were found at the same time at several sites in the Huyck Preserve. Specimens of larvae, pupae, and adults of both species have been deposited in the Museum of Comparative Zoology in Cambridge, Massachusetts.

\footnotetext{
${ }^{1}$ I am grateful to the directors and staff of the E. N. Huyck Preserve for generously supporting a summer of research, and to Dr. R. Dalgleish for help in obtaining financial support for publication of this paper. I am also indebted to Dr. R. Gagné for identifying adult specimens, Dr. F. Shaw for useful advice, and Dr. M. Gordon for supplying culture media. My wife Mary Jane has offered valuable help with all stages of this study. Photographic supplies were furnished by Harvard University.

${ }^{2}$ Present address Depto. Biología, Universidad del Valle, Cali, Colombia. Manuscript received by the editor September 25, 1970.
} 
Precopulatory behavior of males

Male flies searched out female pupae ${ }^{3}$ which were suspended on silk threads, then hung under them (Fig. I) until the adult emerged. They flew in a characteristic bobbing pattern as they searched for pupae hanging under the bottom surfaces of logs. Usually, as they flew along the length of the $\log$, they bobbed up until they hit the bottom of the log with their front legs, which they carried raised over their heads, then flew down and onward. Sometimes they swung back and forth from one side of the $\log$ to the other as they flew along under it. They made frequent darting flights to other objects in the vicinity where they occasionally landed and paused.

Although the bouncing flight appeared erratic, the pattern is probably controlled. A male which was confined in a cage which had pupae fastened to the roof with long pins was able to touch pupae over and over without running into pins.

The males' responses to pupae they encountered are listed below in order of increasing attention to the pupa.

I. no change in flight pattern

2. hover near pupa monmentarily, back off

3. (often preceded by hovering) touch pupa, back off

4. touch pupa, stop flying and cling to it, move about under it, fly off

5. same as 4 except end by hanging immobile under pupa with ventral side of thorax pressed against the dorsal surface of the pupal thorax (Fig. I)

Encounters with a given pupa did not always elicit the same reaction, even from the same male.

The males probably used either visual cues or tactile cues from their front legs (or both) while locating pupae during bobbing flight. The males usually mounted a pupa with their anterior ends at the pupa's anterior end and their longitudinal axes parallel to that of the pupa. Without exception, the anterior end of a pupa hung below the rest of its body, and this position appeared to be the cue used by males in positioning themselves: a pupa which was hung so the posterior end was the lowest was repeatedly mounted backwards.

Males hung persistently only under pupae of females about to

\footnotetext{
${ }^{3} \mathrm{It}$ is possible that the males were actually responding to pharate adults still within the pupal cuticle rather than to actual pupae. Observations were not made on this point, so the term "pupa" below should not be construed to exclude this possibility.
} 


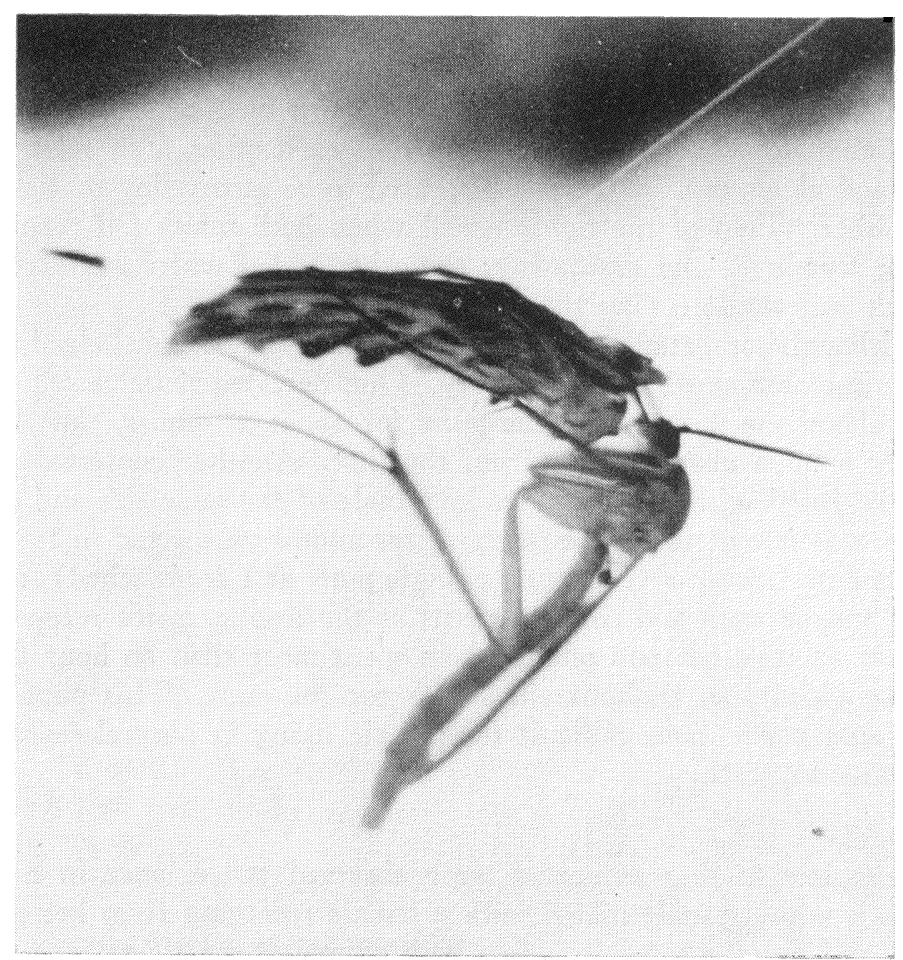

Fig. 1. A male L. subcaeruleus clinging to a pupa.

emerge. Only five of more than 40 pupae observed in nature had males hanging under them. All five of these pupae were females, and four of them produced adults within one day (the average pupal life is about four days). The fifth died. Males often mounted young or male pupae again and again only to climb off each time, but seldom dismounted from mature female pupae. Males also consistently ignored some unsuitable pupae but repeatedly touched and mounted other unsuitable pupae nearby.

There was brisk competition among males for suitable pupae. Males flying near a male hanging under a pupa often bumped against the resident male as they flew by; three extended fights over pupae were observed; and an additional male was found hanging on the back of a newly emerged female which was mating with another male. In each of two fights observed from the beginning, one male 
was hanging immobile under a pupa and a second male approached, bumped against the resident male, and clung to the pupa. Both males held the pupa or the pupal thread with their front and middle legs, and buzzed their wings and pushed each other with their hind legs and abdomens. Each male appeared to be attempting to dislodge the other. Two of the fights ended when both males fell from the pupa, separated, and flew away; the other ended when the intruder, which was smaller than the resident, left.

Although combatants left the pupa when they were dislodged, they sometimes remained in the vicinity. One defeated male rested on a leaf about one meter from the pupa for about a minute, then hung under a pupa about $20 \mathrm{~cm}$ from the first. Another contested pupa was revisited within 15 minutes by a male of the same size and color pattern as one of the contestants. After another suspected fight (two males flew from the vicinity of a single pupa as I approached), males of the same sizes and color patterns as the fleeing males reappeared within 15 minutes, and one of them spent more than an hour flying in the vicinity in an apparent search for the pupa. This pupa was in a spot particularly difficult for a male flying in normal searching patterns to reach.

\section{Mating}

Complete mating sequences were observed twice, once in nature when a waiting male mated with a female emerging from her pupal skin, and once in captivity when both animals were flying in a cage. In the first case, the female's thorax and head (the lowest parts of her body) were cradled against the ventral surfaces of the male's thorax and coxae as she emerged from her pupal skin, and he maintained this contact by lowering his body as she emerged. As she came free from the skin, the female drew her legs free from the pupal cuticle, then extended them perpendicular to her body axis just as other emerging adults did (below). However, in contrast to others, her abdomen came completely free from the pupal cuticle very quickly, and for a moment she lay just above the male, their bodies both horizontal. Almost immediately he curled the tip of his abdomen over the tip of hers (he had curled his over the tips of her wings twice before her abdomen emerged) and pressed it against the ventral surface of the tip of her abdomen, then lowered his abdomen until they were both nearly vertical, and gently turned her $180^{\circ}$ on her longitudinal axis with his legs. This brought her legs against his legs and the pupal skin, and she clung to them weakly. Both animals remained nearly motionless in this position (Fig. 2) 
for about three hours. Then after a few twitches by the female, they separated, and both hung for several moments under the pupal skin.

The other observed mating involved two individuals placed in a cage after being captured in copulo under a pupal skin and then kept separated for several hours. In the course of several minutes of flying around the cage, the male brushed against the female several times as she sat on the floor, and each time she started and moved away. One time, however, she did not move away when the male landed on her back. He immediately curled the tip of his abdomen over hers, engaged it against the ventral surface of the tip of hers, and then dismounted and turned $180^{\circ}$ to face away from her. They both rested on the floor for over an hour, then separated. On another occasion a male attempted to mate with another female (a virgin) in the same situation, but was unsuccessful. Although she allowed him to land on her back, she did not let him press the tip of his abdomen against the ventral surface of hers: whenever he curled the tip of his abdomen over hers, she moved her abdomen to the side or pressed it against the surface she was on.

It is not clear how of ten copulation occurs between two free-flying individuals in nature. It may be difficult for males to locate, recognize, and couple with free-flying females, but the smoothness with which the one male mounted, locked, and then turned and dismounted suggests that this type of mating may occur naturally.

\section{Eggs}

Oviposition by both virgin females in captivity and presumably mated females in nature appeared to be nearly identical. Eggs were laid singly rather than in clusters. The female walked quickly over the surface on which the egg would be laid, pausing occasionally and rubbing the tip of her abdomen rapidly back and forth on the surface. Egg laying did not always accompany these pre-oviposition movements. The female bent her abdomen forward underneath her body so that the tip was nearly under her thorax as she laid an egg. Usually she deposited some clear fluid just before laying the egg, and this dried and remained visible around the margins of eggs laid on glass in the laboratory. In captivity, some eggs lacked this fluid, and some deposits of the fluid lacked eggs. The maximum number of eggs laid by a female in captivity was I ro. Each egg was oval, about $0.8 \mathrm{~mm}$ long, and covered with tiny points apparently similar to those on the eggs of L. walkeri (Brocher 193r). Eggs found in 


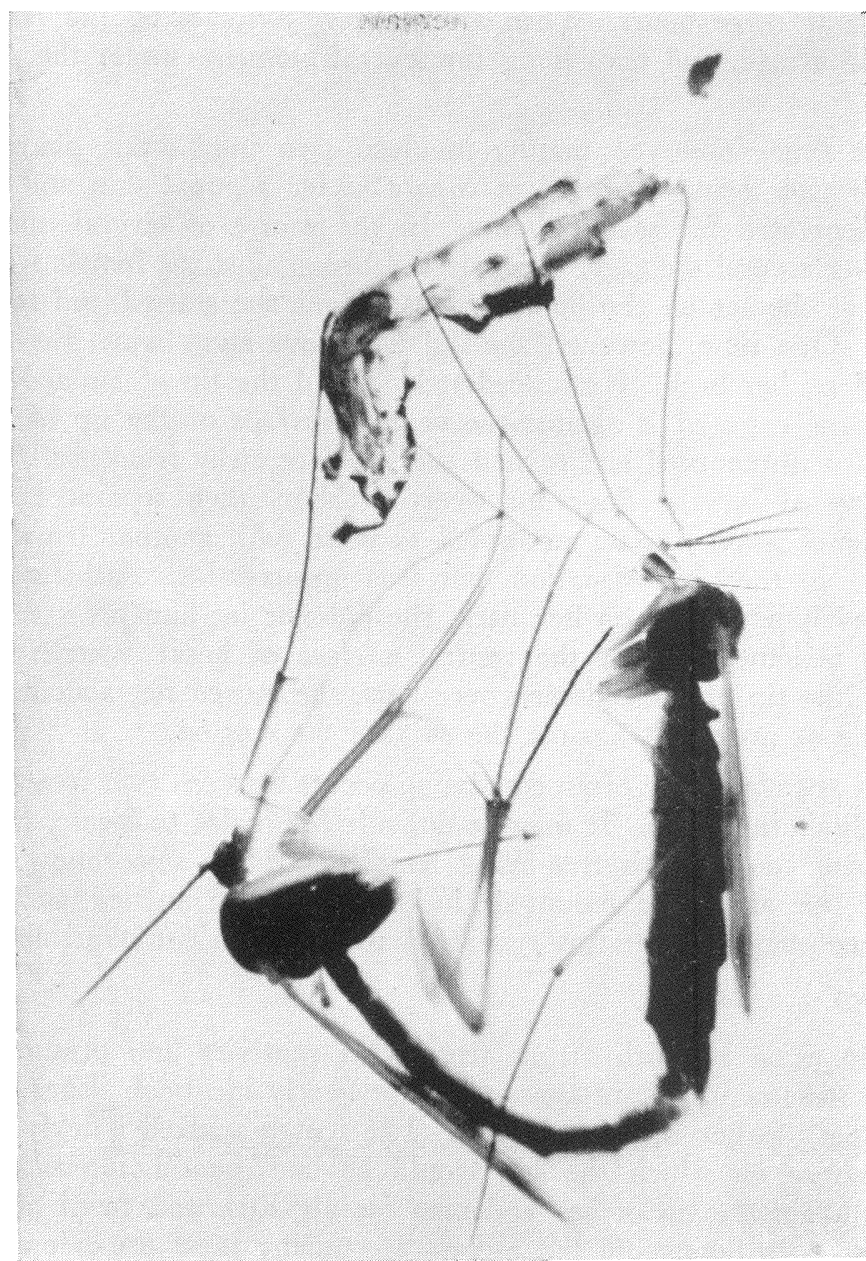

Fig. 2. A newly emerged female (right) and a male $L$. bifasciatus mating under the female's pupal skin. 
nature were on the undersurface of a $\log$ where there were larvae and pupae present, and in cracks between the edges of bracket fungus sporophores and tree trunks.

\section{Larvae}

The larvae found during the study period (I July-I Sept., I968) varied from 3 to $19 \mathrm{~mm}$ in length. They were similar to the larva of Leptomorphus walkeri described by Madwar (1937), with a sclerotized head capsule and 12 fleshy body segments. As in $L$. walkeri, the labrum bore eight prominent papillae, and each maxilla also carried one large papilla. Madwar termed these structures "sensory papillae", and Brocher (I93I) called them teeth ("dents"), but lines of silk were seen emerging from these papillae during spinning activity, so spigot seems a more logical name.

The I2 fleshy segments behind the head capsule were all moist. The color of their cuticle ranged from nearly transparent to light brown in different individuals, with markings of darker brown. The heart was just under the dorsal surface, and in a nearly fully grown larva a peristaltic beat originated at the posterior end and moved forward about 50 times/minute. The larvae were always found upside down under more or less horizontal surfaces such as the undersides of fallen logs or bracket fungus sporophores. Each larva was on the bottom surface of a sheet of silk it had spread under the supporting object. The sheets were relatively flat, and often I $\mathrm{mm}$ or more below indentations in the supporting surface. There was always a dendritic network of slime trails on the silk, and the larvae were always on one of these trails (Fig. 3).

Larval sites had several features in common. There was almost always (one exception in more than 60 cases) an open space of more than $3 \mathrm{~cm}$ (usually much more) between the larva and the nearest surface below; the sites were always at least slightly moist; and the silken ${ }^{4}$ sheets were nearly always built under fungal sporophores. The larvae were found under several types of sporophores, including both brackets (probably Fomes) and others which spread along the surfaces of rotten logs.

Many of the sporophores occupied by larvae appeared to be perennial, having a soft, moist, and evidently sporulating layer during part of the study period, but were dry and evidently not releasing

${ }^{4}$ The material has not been chemically analysed, and is called silk because it is formed in threads and is white. 


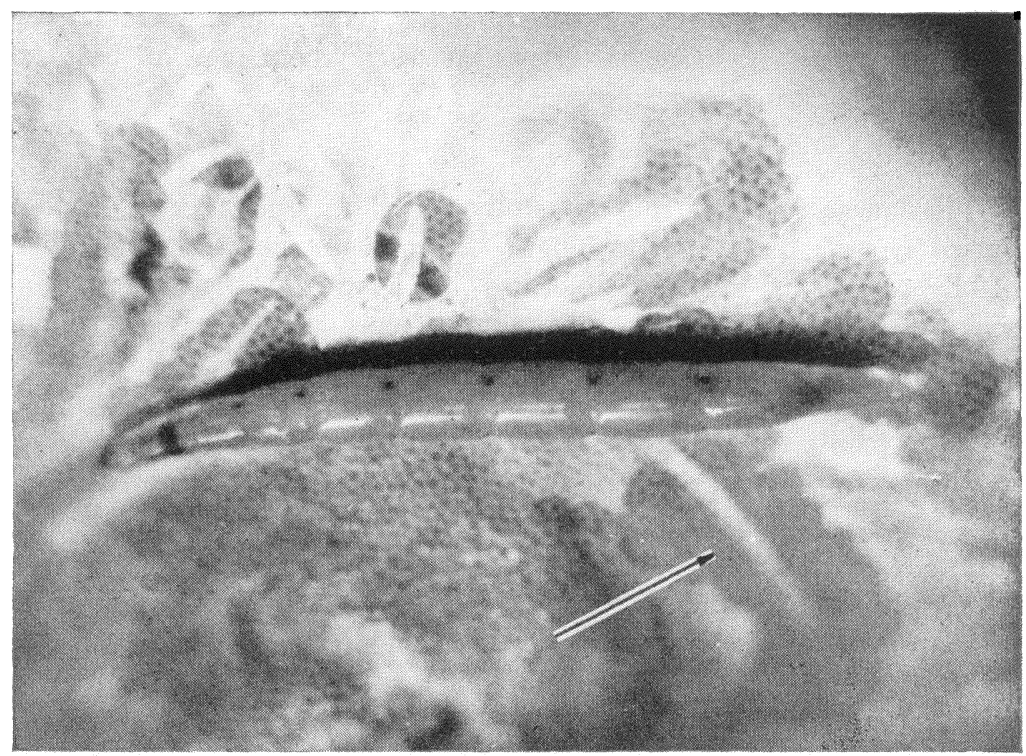

Fig. 3. A L. subcaeruleus larva on its silk sheet. Note the slime trails and the areas where the larva fed recently (arrow).

spores at other times. Although pupae, pupal skins, fragments of silken sheets, and (occasionally) dead larvae were found under apparently dormant bracket sporophores, living larvae were found only under active brackets. Sporophores on the undersurfaces of fallen logs sometimes had large "dormant" areas adjacent to active areas, and larvae were always under only the active areas.

The silken sheets under which the larvae lived often contained large numbers of tiny specks barely visible to the naked eye. When pieces of sheet which had specks in them were placed on a sterile nutrient medium (Wort agar), fungus grew, but no fungus grew when the same medium was inoculated with pieces of speckless sheet spun by captive larvae on surfaces which lacked fruiting fungi. Thus the specks which were common in sheets in nature were probably fungal spores.

Undisturbed larvae under sheets of silk were observed in only four activities: resting motionless, moving across the sheet, eating holes in the sheet, and laying silk. Larvae spent the majority of their time both day and night motionless.

The larvae could move forward and backward, in each case by 
means of peristaltic contractions whichs originated in the trailing segment(s) and moved toward the leading end. A band of slime was laid on the silk when one of the fleshy segments came in contact with it, so as the larva moved forward, it left a slime trail behind it. A dendritic network of slime trails extended to all parts of the sheet, and the larvae always followed trails as they moved about. A peculiar method of turning around (also noticed in the mycetophilids L. walkeri by Madwar 1937, Platyura nigricornis by Mansbridge and Buxton 1933, and Sciophila sp. by Osten Sacken I886) enabled them to remain on a slime trail at all times without constructing any circular trails: they turned the head $180^{\circ}$, and crawled back along themselves, advancing all segments simultaneously so that the head moved along the back or side in what had been the posterior direction while the tail continued to move forward until the turn was complete (see Fig. 4e). The function of the slime trails is not clear.

While spinning and feeding, the larvae moved their anterior four segments without moving the rest of the body. Larvae ate holes in their sheets by moving their heads slowly in arcs in front of themselves. The sheet was broken where the head touched it, and the portion inside the cut was ingested as the head swung. A part of the head capsule (the mandibles?) moved rapidly as the silk was cut. The rapidity with which the silk disappeared suggests the larvae probably used a silk-digesting enzyme. The alimentary canals of several dissected specimens were full of spores but contained no visible traces of any other solid matter, indicating that the larvae usually ingest nothing other than their sheets and their contents and rapidly digest the silk.

Usually a larva immediately filled each hole it made in the sheet, swinging its head and segments I-4 rapidly from side to side in a complicated pattern (see below). A new mat of silk lines running in several directions across the hole in the sheet was produced by these spinning movements. The new lines were almost always attached to the edges of the hole in the sheet rather than to the surface of the fungus. Silk eating was always preceded by a semicircular sweep (see description of spinning movements below), and was always followed by spinning activity. Semicircular sweeps were not always followed by eating, however, and larvae on sheets often spent periods of time spinning but not eating.

A larva often concluded a series of spinning movements by placing its anterior end against the sheet and moving its whole body back- 

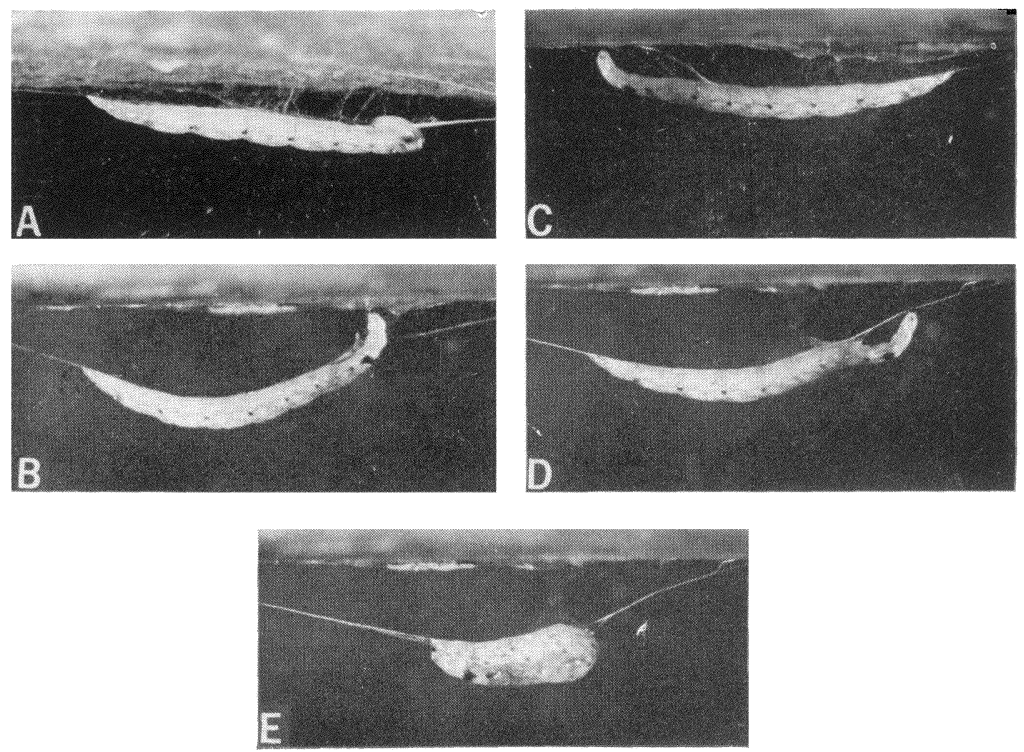

Fig. 4. Construction of the pupal line. Activities illustrated are swinging $(a)$, cutting $(b)$, tapping $(c)$, trimming $(d)$, and turning around $(e)$.

wards, leaving a slime trail extending all the way to the end of the new silk. Larvae left overnight on a new surface often constructed long dendritic networks of silk trails, each with a slime trail running down the middle.

The larvae were apparently able to perceive vibrations in their sheets, but either fled from or did not respond to the struggles of animals such as sminthurid collembolans caught in the sheet. They usually appeared to sense the presence of another larva before contact was made, and often moved away as another approached, but occasionally when they met head to head, larvae fought. Their heads locked, and they swung from side to side in wide arcs, sometimes quite violently. After a few swings they disengaged, and one or both backed away.

Construction of the pupal line

Before pupating, the larvae always suspended themselves on a line under or near the surface where they had been feeding. The process of construction of this line was nearly identical in each of four cases observed in captivity. 
Larvae maintained apparently normal activity until just before they began to construct the pupal line. When four larvae were placed on a $\log$ in an observation chamber, two individuals which were to pupate 12 hours later could not be distinguished on the basis of the rate or type of their activity from the two which did not pupate.

A series of probing movements (observed only once but probably performed in each case) immediately preceded line construction. The larva extended the anterior half of its body downward, perpendicular to the log's surface, and waved it from side to side. It repeated this behavior several times, each time after raising itself back to the log's surface and moving forward a centimenter or so. This probing behavior may be used to select appropriate pupation sites, informing the larva of any objects close below it. Pupae were never found where any such object could interfere with them as they swung on their lines.

The construction of the pupal line took about 40 minutes. The larva first added silk to a long, relatively straight trail, and cut the silk along the edges of this trail, leaving only the central portion intact. It laid silk with bursts of a swinging motion which was less complex than the silk-laying movements described below. The larva's head moved from the slime trail to one ventrolateral surface of segment 5 or 6 (abdominal segment 2 or 3 ) of its body (Fig. 4a), back to the trail, and then to the other side. Bursts of swinging often lasted 15 seconds or more, and the larva inched forward following each burst.

Larvae often cut silk just after moving forward: the head bent to the side of the trail, and moved slowly (usually forward) in a line nearly parallel with the trail. The threads encountered by the head were severed, and the central portion of the trail was progressively freed from the log's surface and sagged down under the larva's weight (Fig. 4b).

At the ends of the trail, where the pupal line would be anchored to the $\log$, the larva spent periods of up to 40 seconds in another activity, tapping its head rapidly against the wood (Fig. 4c). The taps were occasionally interrupted by swinging silk-laying movements. These swings occurred only singly and infrequently early in a burst of tapping, but occurred more often and in longer bursts toward the end. Tapping sometimes covered a relatively large area, and the pupal line was often split near its end, with each smaller line running to a different area of attachments. This pattern probably 
functions to anchor the pupal line more securely by allowing an increased area of attachment.

One other kind of behavior occurred on portions of the trail which hung free of the log. The larva drew its head slowly along the line (Fig. 4d), moving its mouthparts rapidly and apparently either packing the strands together or eating away some strands of silk. These "trimming" movements often resulted in a perceptible thinning of the line.

The four types of behavior - swinging, cutting, tapping, and trimming - were interspersed throughout most of the construction of the pupal line. Cutting and tapping were more frequent during the early stages, and most of the trimming which occurred during this time was done as the larva backed slowly away from a tapping (attachment) site. The larvae were able to turn around on the pupal line by crawling back on themselves just as they did while on their sheets (Fig. 4e), and occasionally ceased work at one end of the line, turned around, and began different behavior somewhere else on the line.

When the line was completely or nearly completely free from the sheet, the larva positioned itself near the center and made final spinning movements which lasted two to three minutes. These movements differed from the swinging movements of line construction only in that the silk was now laid from the pupal line to a dorsal section of the larva's body. The head swung farther and farther over the back, eventually crossing the dorsal midline of segments 5 and 6 with each swing. This behavior produced a small collar of silk which anchored the larva's body to the pupal line (arrow in Fig. 5a). Larvae inched themselves forward along the line as they spun the collar; the distances they moved varied, and thus the number of segments anterior to the collar and free of the pupal line varied.

The larva trimmed the line near its head for a short time after fastening itself into position on the pupal line, but soon the head movements diminished, and the segments anterior to the collar sagged away from the line and hung below the rest of the body. Segments 4-12 began a series of contractions, and segments $I$ and 2 (thoracic segments I and 2) swelled. The contractions occurred approximately every I 5 seconds at first, but soon slowed considerably. As segment I expanded, it began to engulf the head capsule, and the larva flexed the capsule ventrally during the contractions so that its ventral surface lay against the ventral surface of segment I (Fig. 5a). 
About an hour after the collar was completed, the body contractions, still accompanied by movements of the head, had decreased in amplitude and slowed to about one every seven minutes.

The contractions and subsequent changes in body form apparently follow construction of the pupal line and collar regardless of the larva's situation. One larva fell off its line as it constructed a collar, and although it began to lay a trail of silk soon after it landed, its anterior end was swollen and it was nearly immobile 30 minutes later.

Although most pupae found in nature were under or near sites where larvae had apparantly fed, the positions of some pupae suggested that larvae sometimes moved several centimeters from their feeding sites before pupating. In one case, seven of eight pupae found on a fallen beech tree were under bare bark at the bottommost surface of the log, several centimeters from the nearest fungal sporophores (brackets on the side of the $\log$ ). Pupae were also usually oriented so that their longitudinal axes were nearly parallel to the longitudinal axes of the logs they were under. The axes of I7 of 21 pupae were more nearly parallel than perpendicular to the axes of the logs they were under. The cues used to achieve these orientations and the functions of the positioning are not known.

Larvae often pupated soon after being brought into the laboratory, and it appeared that cues from the environment may sometimes induce them to pupate prematurely. In one typical case, 24 bracket fungus sporophores inhabited by larvae were removed from beech trees and pinned to cardboard boxes in the laboratory. Within two days the sporophores became noticeably drier, and eight of the largest larvae constructed pupal lines and pupated. None of the other larvae pupated, and all except two which were on surfaces particularly well sheltered from air movement were dry and dead in the midst of their sheets after five more days. On the other hand, some larvae kept on portions of sporophores in a cool (about $15^{\circ} \mathrm{C}$ ) and moist environment in captivity only pupated after more than a week. Results like these suggested that once a larva reaches a certain size or maturity, it will pupate if its food source dries up.

\section{Pupation}

Larvae remained suspended for about 24 hours before they pupated. At the end of this period, segments $I$ and 2 swelled even more, so that the line dividing them became less distinct and the head capsule was nearly completely engulfed (Fig. $5 \mathrm{c}, \mathrm{d}$ ). Hints of ridges also 

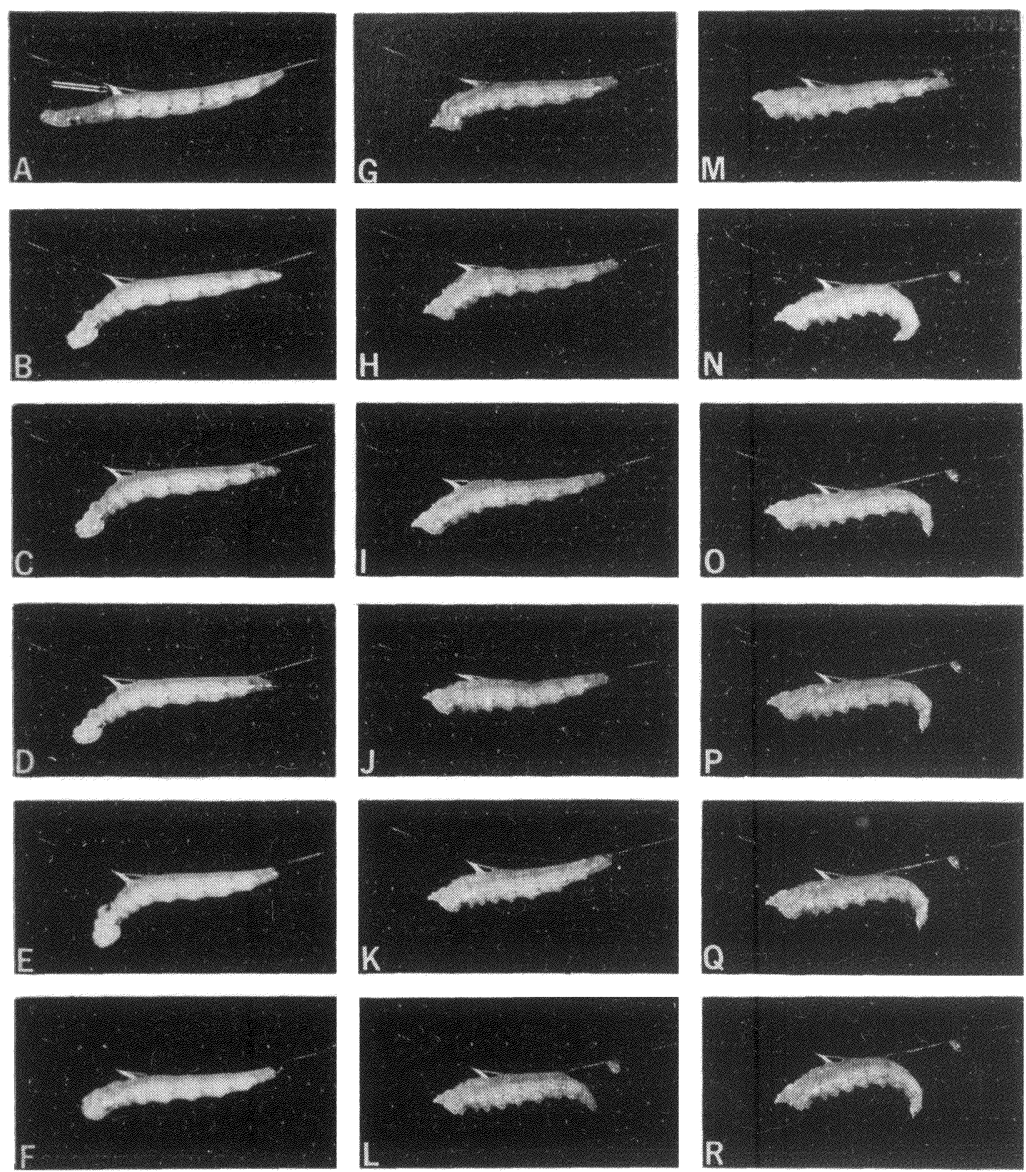

Fig. 5. Pupation. Pictures $a, b, c$, and $d$ were taken $20,2,1 / 2$, and $1 / 4$ hour respectively before picture $e$. Strong contractions had just started when $e$ was taken, and each subsequent picture was taken 30 seconds later. Arrow in $a$ marks collar of silk spun by larva. 
appeared along the dorsal and lateral midlines of more posterior segments (Fig. 5 a-d). About 40 minutes before the transformation to pupal body-form occurred, the posterior tip of the larva's body began to darken and shrink in circumference (Fig. 5 a-d).

The transformation to the pupal body-form took only about five minutes. It was observed twice in nature and twice in captivity. Contractions of muscles in segments posterior to segment 3 accompanied the transformation. In two cases there were preliminary contractions causing the body to arch away from the log. Then, in all cases, powerful peristaltic contractions in the posterior end of the body caused it to thin, straighten, and lengthen. These were presumably contractions of circular muscles, and, like the contractions following collar construction, probably served to increase the internal fluid pressure and cause the cuticle to give in weaker places and expand into the form of a pupa.

Almost immediately after the contractions began, segments $\mathrm{I}$ and 2 swelled even more and the head capsule disappeared within them. After less than a minute, the anterior end suddenly assumed a new shape, with two small "horns" (which would house parts of the antennae) protruding (Fig. $5 \mathrm{~g}$ ). About one minute later the cuticle just anterior to the last two and one half segments began to appear loose, and wrinkled during each contraction. Suddenly the rest of the body broke away from these segments, and they were left stuck to the pupal line while the new posterior end of the animal hung free (Fig. 5 1). The contractions continued, and the new tip extended straight behind the body during each contraction (Fig. $5 \mathrm{~m}$ ). After another two minutes, the contractions had shifted the whole animal forward so that the new posterior tip could no longer touch the discarded segments. The contractions were substantially weaker by six minutes after the beginning of the transformation, and the new posterior tip no longer extended straight behind during each contraction but hung below the line in the characteristic pupal attitude (Fig. $5 \mathrm{q}$ ). The contractions continued for several more minutes, but rapidly became weaker and more infrequent, and soon the new pupa hung motionless.

Emergence of the adult

The pupa dried within a few hours after pupation and remained motionless until an adult emerged about four days later. The pupal lives of five individuals kept in captivity at room temperature lasted from three and one half to five days ( \pm 6 hours).

The complete process of emergence from the pupal skin was 
observed twice, and portions of the process were observed several other times. All emergences (except that of a female attended by a male - see above) were nearly identical. First the pupal skin covering the dorsal surface of the thorax and head split, and the adult began to emerge through this hole. The insect was nearly motionless during this process, and its forward movement through the pupal skin probably resulted from peristaltic contractions of its abdomen. About eight minutes after the head and thorax first began to emerge, the legs, which by now were nearly free, began to twitch. The fly gradually pulled them forward and out of the pupal skin, then extended them horizontally so they were perpendicular to its longitudinal axis. The wings came free about four minutes later; at first they were soft and slightly bent, but they soon straightened and began to harden. After about 15 minutes, the entire animal was free from the pupal cuticle except for the terminal segments of its abdomen which were just inside the skin and supported the animal as it hung facing downward. While the fly hung in this position, the diameter of its abdomen decreased, and its new cuticle hardened. After about two hours the fly seized the pupal skin with its legs and swung its abdomen free of the skin. Undisturbed individuals hung motionless in this position for an additional hour or so before flying away.

The mechanisim by which the flies supported themselves with the tips of their abdomens while they hardened was clarified when one fly fell completely free from its pupal skin unusually early. The last two segments of its abdomen were flexed dorsally so they formed a right angle with the rest of the abdomen. Apparently this crook at the tip of the abdomen is wedged tightly enough in the anterior end of the pupal skin that the weight of the fly's entire body is supported.

\section{Discussion}

The observations of pupation indicate that the larval cuticle is not shed prior to pupation, that the last two and one half segments of the larva are discarded, and that the larval head capsule is engulfed by the pupa during pupation - all phenomena apparently undescribed in other Diptera (Imms 1964). It appears that the larval head capsule may migrate the length of the pupa after being engulfed. Head capsules appeared to be emerging from the ventral surfaces of abdominal segment \#8 of some preserved pupae, and were on this surface, completely free of the pupa in others which 
were darker and thus apparently older. More work is clearly needed to establish the details of this remarkable metamorphosis.

It remains possible that the larval cuticle was shed during pupation but was so thin that its movement was not observed; indeed the swelling of the anterior segments prior to pupation suggests a rupture of the larval cuticle. A search for signs of larval cuticle on pupae did not yield clear evidence of its presence: pupal cuticle did have the same number and pattern of spiracles as the larva's, but seemed to lack the rows of dark denticles near segmental boundaries on the ventral surface which were quite prominent in larval cuticle.

Other mycetophilids are generally thought to shed their larval skin during pupation (Osten Sacken I886), but masses of material occur near the posterior tips of the abdomens of the pupae of several species, including Ceroplatus sp. (Malloch I9I7), Macrocera anglica (Madwar 1935), Leptomorphus walkeri (Edwards 1925, Madwar 1937), Speolepta leptogaster (Madwar I937), Mycetophila cingulum (Madwar I937), and Mycomya limbata (Lindner 1949). Although in some cases (e.g. Macrocera anglica) this material apparently is the larval cuticle (Madwar I935), the details of the pupa's position within its cocoon indicate that the material may not be larval cuticle in at least two of the other cases. Threads are attached to lateral projections along the sides of the pupa of Ceroplatus sp. (Malloch 19I7) and Mycomya limbata (Lindner 1949). As Malloch points out, any silken attachments the larva might make to itself would be shed with the larval cuticle, and it is difficult to imagine how the pupa could be suspended in such a way if the larval cuticle is shed. It thus seems possible that the larval skin is not shed in these species, and that the mass of material behind the pupa is a discarded portion of the larva's body.

The selective advantages of some aspects of Leptomorphus pupation are not clear. Ingestion of the head capsule and the possible failure to shed the larval skin may be involved in maintaining silk attachments to the pupa, and thus in suspending it on a thread. Suspending the pupa may in turn be advantageous at least in females because it makes the pupa more easily found by flying males. The contents of the discarded larval segments and the function of discarding them are not known. Active larvae defecated repeatedly, and their feces were a much lighter brown than the discarded body section, 
indicating that disposal of this section does not represent elmination of waste from the digestive tract.

Leptomorphus walkeri, the only other member of this genus whose natural history has been described, appears to be similar to the species of this study. The form of the eggs and the sites at which they occur are nearly identical to those described above (Brocher I93I). The larvae are also associated with fungi (Edwards 1924, Brocher I93I), usually on the undersurface of the fungus (Brocher I93I). They probably also build silk sheets: Edwards (1924) notes that they build a "web", and Brocher (I93I) reports that they lay networks of silk trails which they use to support themselves under the fungi, but Madwar (1937) claims that they construct only slime tubes. Brocher found that larvae sometimes ate silk from old trails, and his brief description of silk-laying movements indicates they are at least roughly similar to those of $L$. subcaeruleus and $L$. bifasciatus. The larval food has not been established, although Madwar (1937) speculated that larvae fed on "microscopic bits of fungus" dislodged when the head tapped against the fungus. The pupae are suspended on threads (Brocher 1931, Madwar 1937), at least sometimes at sites with open spaces below (Brocher I93I).

These observations indicate that the larvae of $L$. subcaeruleus and $L$. bifasciatus normally feed on spores caught in their sheets: they were almost always found under spore-shedding structures of fungi; they constructed sheets of silk which collected spores; they periodically ingested these sheets and their contents; and their alimentary tracts contained large quantities of spores. Other mycetophilid larvae, in the genus Ceroplatus, apparently subsist on spores caught in sheets of slime (Madwar 1937, Stammer 1937), but are carnivorous at times (Mansbridge 1933). Several Leptomorphus larvae were found during this study which were not near fruiting fungi, and one dead larva was partially eaten by two other larvae in captivity, indicating that these species of Leptomorphus may also be carnivorous at times.

Fig. 6. Spinning motions of L. subcaeruleus larvae. The attachments made by one larva in a typical series of swings are represented by dark bars in $A$. The sequences of successive attachments during four typical series of swings by two individuals are shown in $B$. The frequency with which an attachment in one zone (labelled in $A$ ) was followed by an attachment in another zone is indicated by the width of the arrow running from the first to the second zone. 

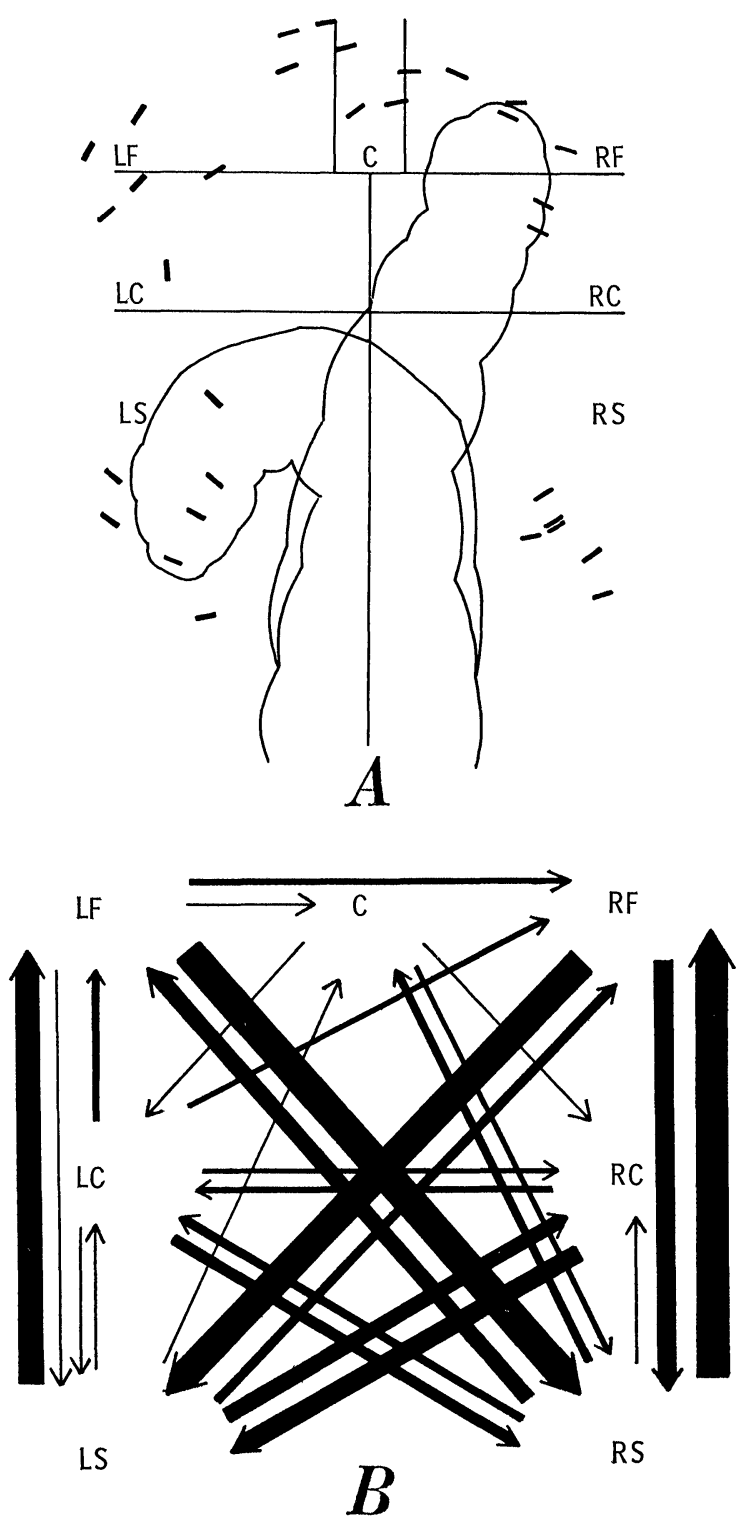
The only other mycetophilid species in which the larvae have been described pupating while hanging on threads in exposed places are Arachnocampa luminosa (Hudson 1950, Richards 1960), and Speolepta leptogaster (Edwards I924). Competition between males for female pupae has been recorded in A. luminosa. Males of this species commonly rest on female pupae, and at least in some situations where there are dense populations, there are often several males clinging to a female pupa which is about to produce an adult (Richards 1960). In contrast to L. subcaeruleus and L. bifasciatus, which probably use tactile or chemical cues to discriminate mature female pupae from others, $A$. luminosa males probably use vision: female pupae luminesce when touched, and glow especially brightly when the adult is about to emerge (Richards 1960). Emerging adults of this species sometimes hang from the pupal cuticle by the tips of their abdomens for several hours (Hudson 1950), and may use the same mechanism to support themselves as the species in this study.

There is probably selection in Leptomorphus bifasciatus and $L$. subcaeruleus favoring males which are able to find, recognize, and hold mature female pupae, and also selection favoring females which pupate at easily located sites. Males which are larger, and thus perhaps more successful in fights, and which copulate long enough to insure the female is fully sclerotized and able to avoid the advances of other males might also be favored. These types of selection probably do not occur in many other Mycetophilidae, since most of the genera in the subfamilies Ditomyiinae, Bolitophilinae, Diadocidiinae, Ceroplatinae, and Mycetophilinae pupate within fungi, under ground, or in cocoons in cracks (Edwards 1924, Madwar 1937). The adults of at least some species, including Phronia sp. and Mycetophila sp. (Colyer and Hammond 195I) form swarms where mating may occur.

\section{Summary}

The larvae of Leptomorphus bifasciatus and L. subcaeruleus live on sheets of silk which they build under fungal sporophores, and apparently subsist on fungal spores caught in these sheets. Pupation occurs while the animal is suspended on a line constructed by the larva, and involves discarding the last two and one half segments of the larval body, but possibly not the larval cuticle. Adult males search for pupae, and rest on female pupae which are about to produce adults. There is brisk competition between males for such pupae. The selective advantages of some of these habits are dis- 
cussed, and comparisons are made with the natural history of other mycetophilids.

\section{Appendix}

Details of larval spinning behavior

The spinning behavior of Leptomorphus subcaeruleus larvae was complex, and it is likely that an evolutionary series of behavior may eventually be established between this species and others whose spinning behavior is less elaborate. The following description, derived from study of $24 \mathrm{f} / \mathrm{sec}$. movies of two $L$. subcaeruleus larvae laying trails on the undersurface of clean saranwrap, will hopefully serve as a basis from which to start such a study.

Silk laying involved three steps repeated over and over: the larva swept slowly once or twice in an often nearly semicircular arc in front of itself with its outstretched head, laid silk by swinging its head quickly from side to side, placed its anterior end against the silk and moved forward, and swept slowly again. Larvae usually tapped their heads on the surface on which they moved several times during one of the slow sweeps, but occasionally a larva did not touch the surface at all. No silk was laid during sweeping activity since there were no lines running in the direction of the semicircular sweeps on glass slides on which larvae had moved. Sweeping motions were much slower than swinging movements: one larva averaged about 0.7 seconds for sweeps of about $120^{\circ}$, but took only $0.04-.08$ seconds to swing approximately the same distance while spinning silk. Larvae usually modified sweeping motions (often by repeating the sweep) when they encountered an object such as another larva's body during a sweep. It appears that sweeping is a type of exploratory behavior, probably functioning to guide the animal as it moves.

Direct observations of active larvae and examinations of glass slides on which they had moved showed that silk was spun during the swinging movements following sweeps. Eight or (often) fewer equally spaced threads of equal diameters were laid down with each pass of the larva's head, indicating that silk from the spigots on the labrum was laid during swinging. Additional threads with smaller diameters were also laid. The threads were attached to the slide near the edges of the silk trail, and the attachments appeared to consist of unpolymerized thread material: each line, whose diameter was uniform between attachments, spread out into a long "puddle" at each attachment. 
In photographing spinning behavior, a movie camera was focussed in the plane of the saranwrap under which the larvae moved, and in analysing the films, I assumed that the movie frames with the larva's head in focus represented moments when the larva was making an attachment, while those with it out of focus represented moments when the head was lifted away from the surface and moving toward the next attachment site. This assumption is supported by the fact that attachments of lines laid on glass slides occur in the same areas as the presumed attachments in the movies.

As illustrated in Fig. 6a, attachments were not restricted to certain areas, but were spread along the sides and in front of the larva. Their distribution was not uniform however: they nearly always occurred near the edges of the spinning area, and were less common in the central portion of the area than in the anterior and posterior portions. The attachments in the central portion were usually made in the later part of a sequence of swings.

There were discernible patterns in the sequences of attachments, but there were also many variations on these patterns. The most common patterns, illustrated in Fig. 6b, included movements from the anterior to the contralateral posterior zone (e.g. RF to LS), and the posterior zones to both ipsi- and contralateral anterior zones (e.g. LS to LF, LS to RF). Larvae often made series of attachments to alternate sides, but also made series of attachments on the same side. The functional significance of these patterns is not known.

The result of the variety of sequences of attachments and the dispersion of attachment points was that a larva laid lines running in many different directions each time it executed a series of swings. Another result was that there were usually no large holes in the array of newly laid threads. The variation in site and sequence of attachments may serve to eliminate holes in the sheet, and thus to prevent loss of spores through these holes.

The variation in movements and the dispersion of attachments may be the product of regular patterns of spinning behavior, but such patterns were not noticed in this short study.

\section{REFERENCES}

Brocher, F.

1931. Observations biologiques sur la larve du Delopsis aterrima Zett. et sur celle du Leptomorphus walkeri Curt. (Diptères mycétophiles). Rev. suisse Zool. $38(4):$ 67-76. 
Colyer, C. N., and C. O. Hammond

1951. Flies of the British Isles. Frederick Warne and Co., L.t. N.Y. EDWARDS, F. W.

1925. British fungus gnats (Diptera, Mycetophilidae) with a revised generic classification of the family. Trans. ent. Soc. Lond. 1924: 505-670, pls. 49-51.

Hudson, G. V.

1950. Fragments of New Zealand Entomology. Ferguson and Osborn Ltd., Wellington, N.Z.

Iмms, A. D.

1964. A General Textbook of Entomology. Barnes and Noble, Inc., N.Y.

INGOLD, C. T.

1967. The Biology of Fungi. Hutchinson Educational Ltd., N.Y.

LINDNER, E.

1949. Die Fliegen der Palaearktischen Region, Band I. E. Schweizerbart'sche Verlagsbuchhandlung, Stuttgart.

MADWAR, S.

1935. The biology and morphology of the immature stages of Macrocera anglica Edwards. Psyche 42: 25-34.

1937. Biology and morphology of the immature stages of Mycetophilidae (Diptera, Nematocera). Philos. Trans. Roy. Soc. Lond. B 227: 1-110.

MaLloch, J. R.

1917. A preliminary classification of Diptera, exclusive of Pupiparia, based upon larval and pupal characters, with keys to imagines in certain families, Part I. Bull. Ill. State Lab. Nat. Hist. $12(3)$ : 161-407, pls. 28-57.

Mansbridge, G. H. and H. W. Buston

1933. On the biology of some Ceroplatinae and Macrocerinae (Diptera, Mycetophilidae) with an appendix on the chemical nature of the web fluid in larvae of Ceroplatinae. Trans. Roy. Ent. Soc. Lond. 81: 75-92.

Osten SAcken, C. R.

1886. Characters of the larvae of Mycetophilidae, printed by the author, Heidelberg: 1-29 (reprinted from Proc. Ent. Soc. Phila. 1862).

RichaRds, A. M.

1960. Observations on the New Zealand glow worm Arachnocampa luminosa (Skuse) 1890. Trans. Roy. Soc. N.Z. 88(3): 559-574, pls. 27-38.

STAMMER, H. J.

1937. Zur Biologie und Anatomie der leuchtenden Pilzmückenlarve von Ceroplatus testaceus Dalm. (Diptera, Fungivoridae). Z. Morph. Oekol. Tiere 26(1-2): 135-146. 

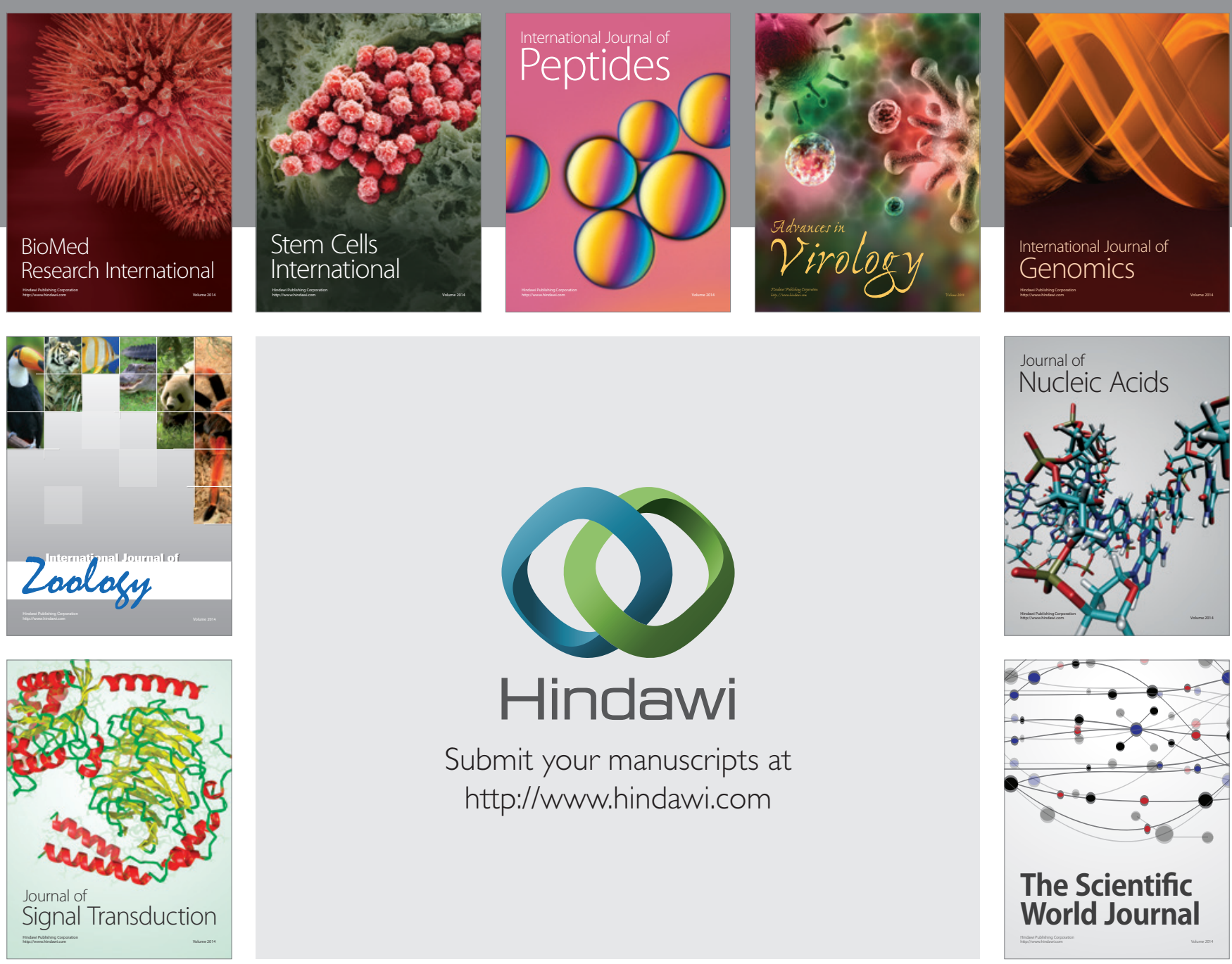

Submit your manuscripts at

http://www.hindawi.com
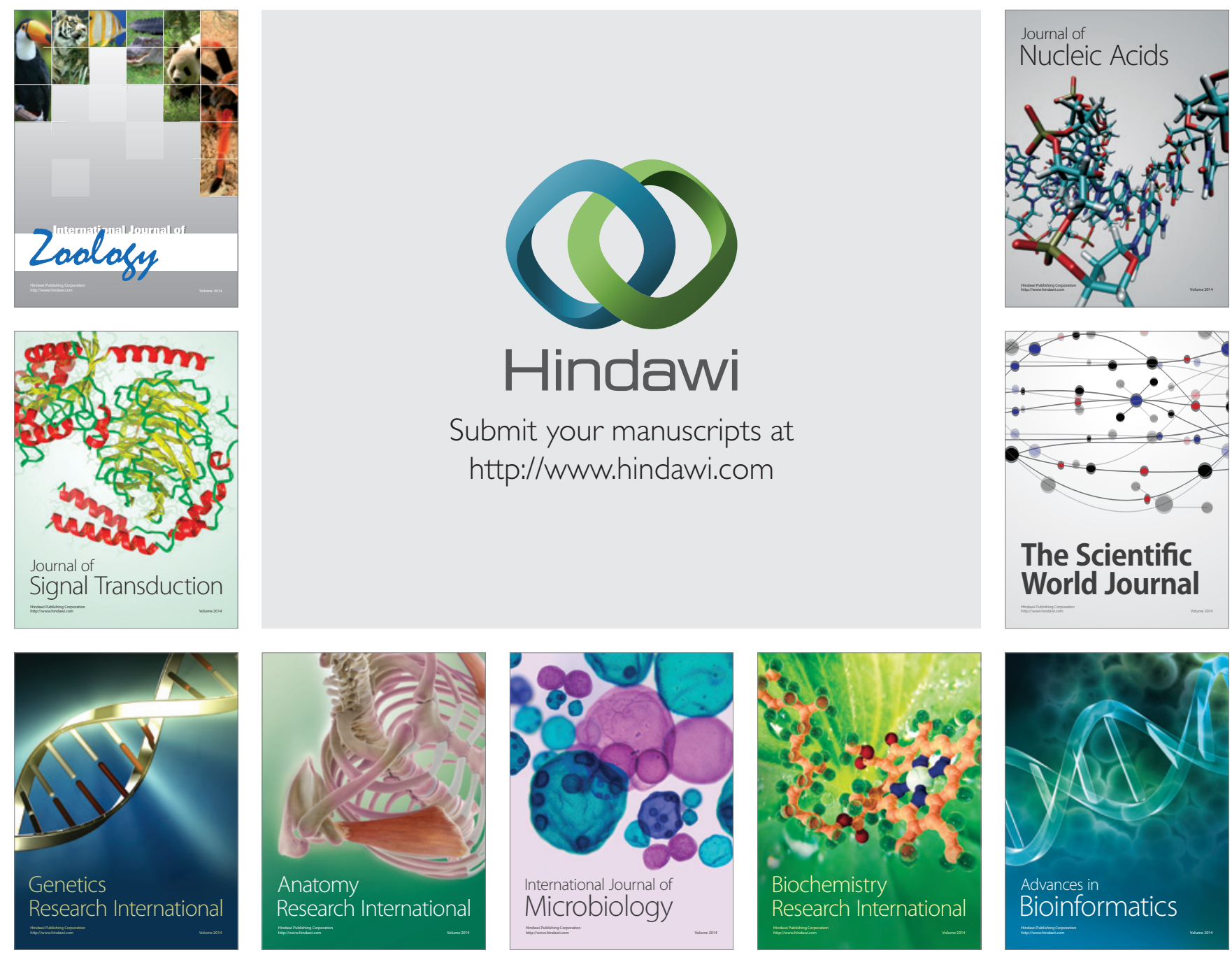

The Scientific World Journal
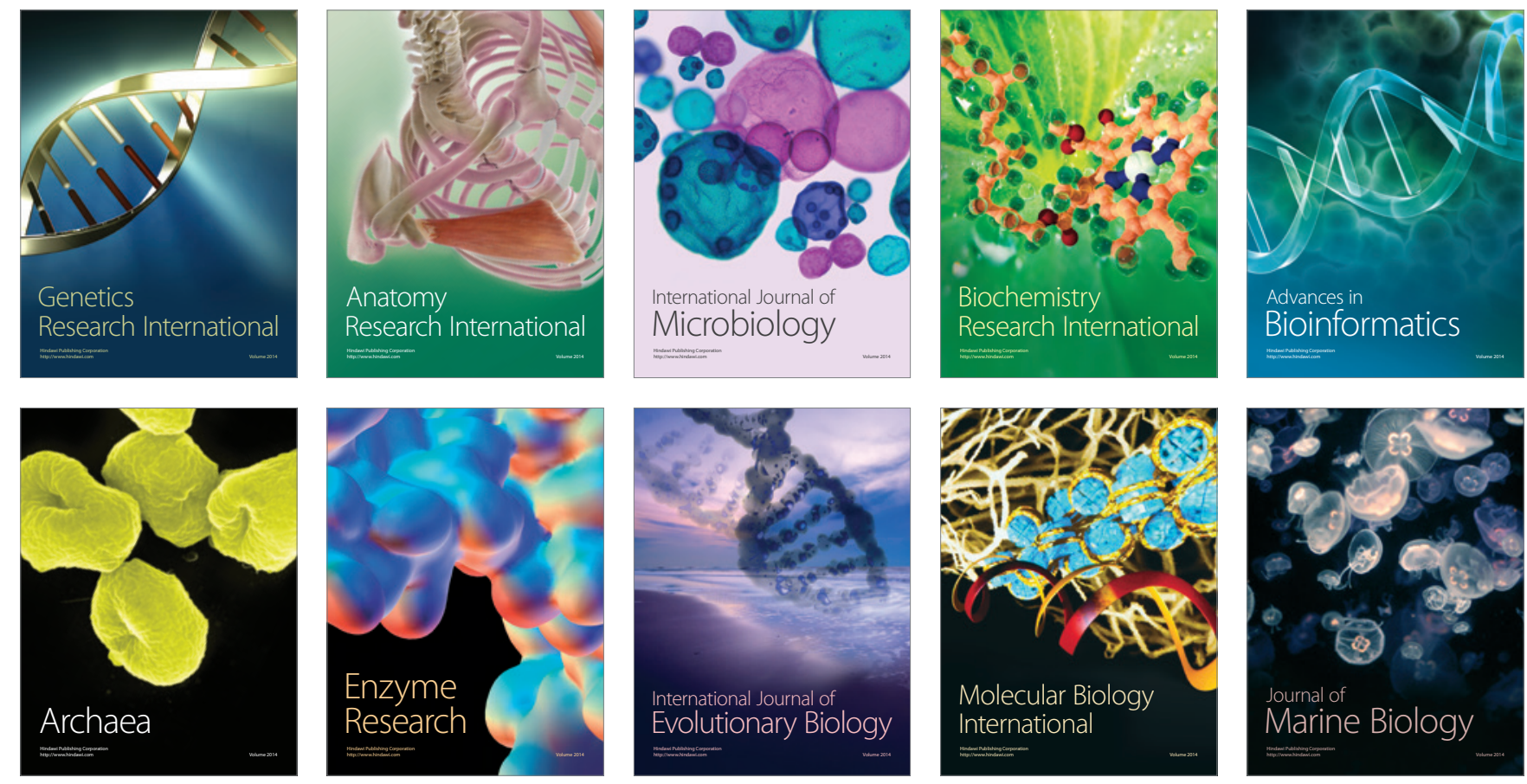